\title{
Extremely Vibrant Routing Scheme for Mobile Adhoc Network
}

\author{
S. Syes Abdul Syed ${ }^{1}$, T. Senthil Kumaran ${ }^{2}$ \\ ${ }^{1}$ Research Scholar, Department of Information Technology, AMET University, Chennai \\ ${ }^{2}$ Associate Professor, Department of Computer Science,ACS College of Engineering, Bangalore
}

\begin{tabular}{l}
\hline \hline Article Info \\
\hline Article history: \\
Received Oct 23, 2017 \\
Revised Dec 29, 2017 \\
Accepted Jan 20, 2018 \\
\hline Keywords: \\
Delay \\
Link Failure \\
Link Residual Life \\
Overhead \\
Velocity
\end{tabular}

\begin{abstract}
This paper aims to improve the performance of the traditional routing protocol for MANET such as DSR and AODV in terms of delay and overhead. The proposed routing scheme is called as Extremely Vibrant Routing (EVR) which adopts with the highly dynamic environment of MANET. The link residual life is estimated to reduce the link failure before forwarding data through a node. The velocity of the moving mode is considered while choosing the next forwarder node. This enables the EVR to decrease the delay in the network. The proposed routing scheme reduces routing overhead and reduces the delay. This scheme reduces the link failure too. The performance is evaluated by using the simulation results obtained by using NS2 simulator.
\end{abstract}

Copyright @ 2018 Institute of Advanced Engineering and Science. All rights reserved.

\section{Corresponding Author:}

S. Syes Abdul Syed,

Research Scholar, Department of Information Technology,

AMET University,

Chennai.

\section{INTRODUCTION}

MANET is used to exchange the information between the nodes in moving mode. The mobile nodes are connected by wireless links is called as mobile adhoc network. The nodes in MANET are autonomous or independent node. The mobile nodes are transferring the information without the help of any external devices such as routers. Each and every node in MANET is autonomous nodes. They are act as relay nodes to support the transmission of other nodes. The nodes itself act as transmitter, receiver and routers. So, the nodes in MANET called as autonomous nodes. As the topology of the MANET changes dynamically, the MANET is called as infrastructure less network. The link between the nodes also changes dynamically. So, it is hard to transmit the data to the node in a highly dynamic environment. Traditionally, there are several routing protocols developed specifically for MANET such as DSR, AODV, and DSDV.

In that the Dynamic Source Routing (DSR) is outperforms than the Adhoc On demand Distance vector (AODV) routing protocol in terms of throughput. But it is not suitable for the highly dynamic environment. In Thakare et al., (2010), the author compared and evaluated the performance of DSR and AODV by using the random way mobility model as a mobility model [1]. The behavior of the AODV and DSR has studied in Khattak et al., (2008) [2]. In this paper, the author analyses the performance over TCP (Transmission Control Protocol) communication protocol and Constant Bit Rate (CBR) traffic model. The obtained results showed that, the Packet delivery ratio is higher when using TCP and CBR while the delay is high for TCP and low for CBR. With high speed the PDR of AODV is lower than the PDR of DSR. The authors have concluded that the AODV and DSR is outperforms than each other with different traffic patterns.

The performance of AODV and DSR is analysed in the highly dynamic environment like VANET in Som et al., (2012) [3]. The authors detected that, the AODV provides better throughput than the DSR but the packet loss is high for ADOV. In Sapna et al., (2009), the author analyses the performance of AODV and DSR by using Network simulator NS2 with Random way point mobility model [4]. The AODV, DSR and 
DSDV routing protocols are analysed and different parameters are compared in Taksande et al., (2011) [5]. In that, the author said that, all the routing protocols are performed well under TCP connection rather than UDP because of retransmission is not available in UDP. Secure and Efficient Distance Effect Routing Algorithm for Mobility (SE_DREAM) in MANETs is described in [6]. In order to provide the secured data transmission and to reduce black hole attacks Prevention of Co-operative Black Hole attack in Manet on DSR protocol using Cryptographic Algorithm is proposed in [7]. Achieving EE without sacrificing the quality of service $(\mathrm{QoS})$ is increasingly important for mobile devices. We first derive the data rate through zero forcing $(\mathrm{ZF})$ and three linear precodings: maximum ratio transmission (MRT), zero forcing (ZF), and minimum mean square error (MMSE). Performance EE can be achieved when all available antennas are used and when taking account of the consumption circuit power ignored because of high transmit power. The aim of this work is to demonstrate how to obtain maximum EE while minimizing power consumed, which achieves a high data rate by deriving the optimal number of antennas in the downlink massive MIMO system. This system includes not only the transmitted power but also the fundamental operation circuit power at the transmitter signal [8].

\section{PROPOSED WORK}

The MANET consists of autonomous mobile nodes connected by wireless link to exchange the information. As the topology of the network changes dynamically, the link between the nodes also changes frequently. The node transmits the information to the indented destination directly if the destination is in the transmission range of the source node. If the destination is present out of the transmission range in the sense the source node transmit via intermediate relay nodes. The mobile node itself acts as relay node. There are many routing protocols are available for MANET. All the traditional routing protocols are built the route before transmitting the data to the destination. So, there is a chance to occur a link failure in MANET. Due to link failure in the network, the data never reaches the destination. After that, the source node reconstructs the route to transmit the data. It causes delay and routing overhead in MANET. To overcome this, this paper proposes a novel routing scheme is called as Extremely Vibrant Routing scheme (EVR). The EVR scheme additionally uses the link residual life to construct the route.

The EVR select the next forwarder node based on the link residual life and the velocity of the node. In EVR, the source node finds the list of neighbor node. And then chooses the next forwarder node based on the link residual life and the distance to the destination. The source node itself does not know the entire rout to reach the destination. In the proposed scheme, the intermediate relay node is also responsible to reconstruct the route failure. Moreover, there is no chance of link failure in the proposed scheme why because the link residual life is also considered while constructing the route. The delay is reduced in the routing scheme by considering the distance between the current node and the destination node. The proposed routing scheme provides the choice of next forwarder node. The reliability is ensured by reducing the link failure in the network. The following block diagram explains the proposed scheme very well.

Figure 1 explains the concept of proposed routing scheme that is the intermediate process between the source node and destination node to transmit the data. In Figure.1, the source node intends to transmit the data to the destination node. So, initially, the source node finds out the nodes which are in their transmission range to form the neighbor list. First, it checks that, whether the destination node is present in the neighbor list or not. If it is present in the sense, it will forward the data to the destination directly. Otherwise, it searches for the best forwarder node in the neighbor list by using the following way.

The link residual life is defined as the duration at which the link exists between the nodes. The LRL is calculated by using the following formula:

$$
L R L=\frac{\text { Distance }}{\text { Relative Velacity }}
$$

Distance indicates that the neighbor (relay) node needs to move to get out of range of the source node. The relative velocity is used to find the direction of the moving node. The relative velocity is calculated by

$$
\text { Relative Velocity }=\text { Displacement } / \text { Time }
$$

The distance between the node and the destination should be in the decreasing manner to become a next forwarder node. The proposed routing scheme reduces the routing overhead by reducing link failure. The routing delay also reduced in the network. This have been analysed by using the simulation results obtained by the network simulator NS2. 


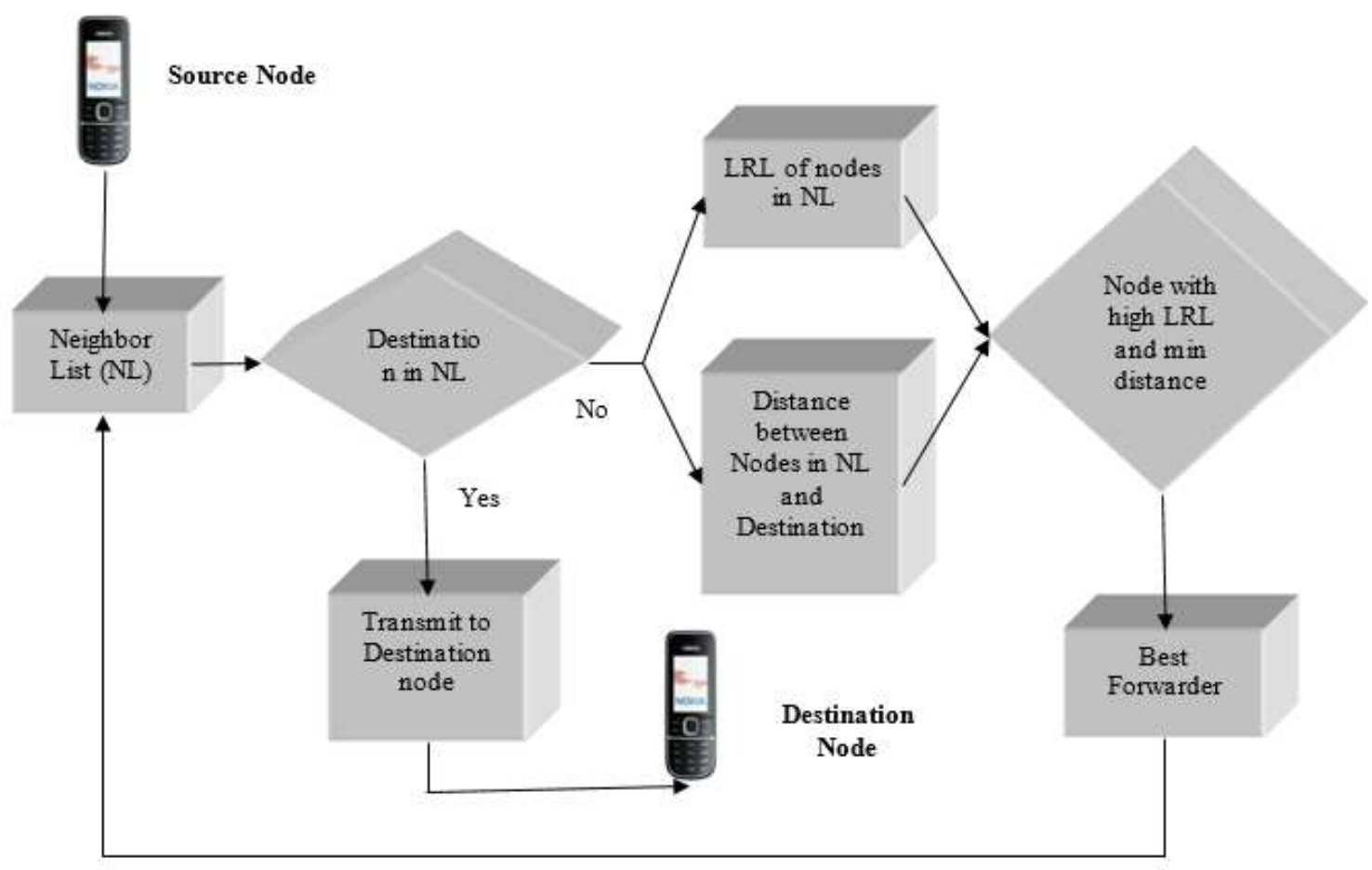

Figure 1. Block Diagram of the Proposed Scheme EVR

\section{RESULT AND DISCUSSION}

The simulation is done by using the simulator NS2. Network simulator is a discrete event time driven simulator. NS2 is open source software which uses C++ and Tool Command Language (TCL) for simulation. $\mathrm{C}++$ is used for packet processing and fast to run. TCL is used for simulation description and used to manipulate existing C++ objects. It is faster to run and change. NS2 is widely used to simulate the networking concepts. The simulation parameter used in the simulation is tabulated below:

21 nodes are distributed in the simulation area $1070 \mathrm{~m} \times 746 \mathrm{~m}$. The mobiles are moving within the simulation area by using the random way mobility model with the speed $5 \mathrm{~m} / \mathrm{s}$. Each and every node has the direct link with the nodes within the range $250 \mathrm{~m}$. The Constant Bit Rate (CBR) traffic model is used to control the traffic flow in the network. The performance or the proposed scheme is analysed by the parameters throughput, Link duration and delay. And the performance is evaluated by changing the mobility model such as Random way point and city section mobility model.

The throughput indicates that the amount of work done per unit time. In the proposed scheme, the throughput indicates that, the amount of data delivered per unit time. Figure 2 demonstrate that the throughput of EVR scheme in MANET. The End to End delay is the average time taken by the data packet to reach the destination. Figure 3 shows the graph plotted between the delays occur in the destination verses simulation time. Lower the delay indicates that the high performance of the proposed scheme. 


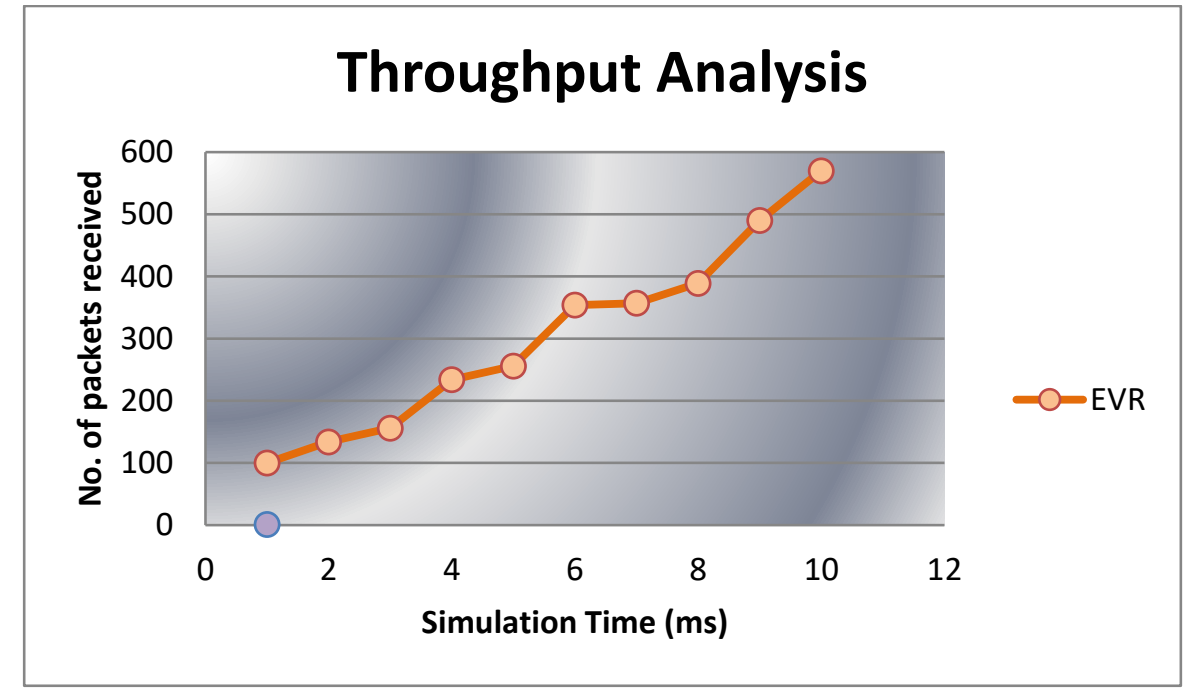

Figure 2. Throughput

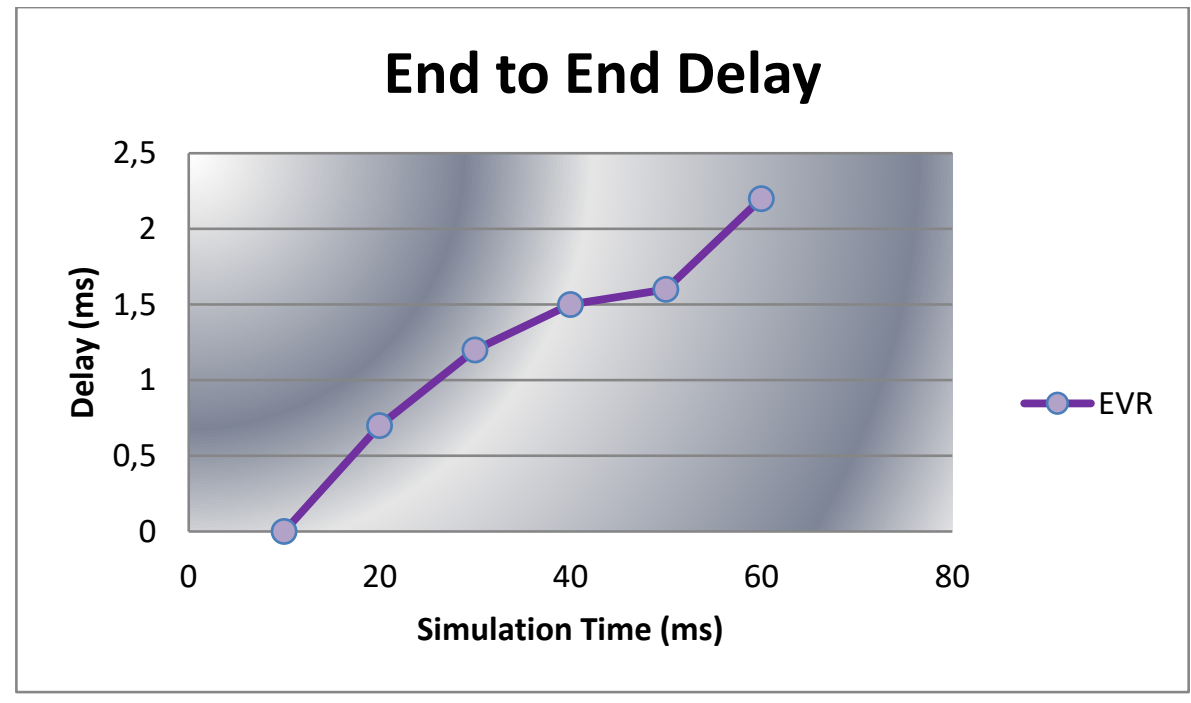

Figure 3. End to End Delay

\section{CONCLUSION}

In this study, a novel routing scheme is proposed to adopt the routing protocol for the highly dynamic MANET. The link residual life and velocity of the moving node plays a very important role while constructing the path to reach the destination. The proposed scheme outperforms than the existing scheme in terms of routing overhead, delay, reliability and link failure.

\section{REFERENCES}

[1] A. N. Thakare and M. Y. Joshi, "Performance Analysis of AODV \& DSR Routing Protocols in Mobile Ad hoc Networks," Indian Journal of Computer Application (IJCA), 2010.

[2] M. A. Khattak, et al., "Challenging Ad-Hoc Networks under Reliable \& Unreliable Transport with Variable Node Density," Journal of Theoretical and Applied Information Technology, 2008.

[3] D. S. Som and D. Singh, "Performance Analysis and Simulation of AODV, DSR and TORA Routing Protocols in MANETs," .International Journal of Recent Technology and Engineering (IJRTE), vol/issue: 1(3), pp. 2277-3878, 2012.

[4] Sapna S. K. and P. R. Desmukh, "Comparison of effectiveness of AODV, DSDV and DSR Routing Protocols in Mobile AD-hoc Networks," International Journal of Information Technology and Knowledge Management, pp. 499-502, 2009. 
[5] Taksande V. K. and K. D. Kulat, "Performance Comparison of DSDV, DSR, AODV Protocol with IEEE 802.11 MAC for Chain Topology for Mobile Ad-hoc network using NS-2 IJCA," 2nd National Conference-Computing, Communication and Sensor Network, CCSN, 2011.

[6] Shanthi H. J. and E. M. Anita, "Secure and Efficient Distance Effect Routing Algorithm for Mobility (SE_DREAM) in MANETs," in Proceedings of the 3rd International Symposium on Big Data and Cloud Computing Challenges, pp. 65-80, 2016.

[7] Vennila G., et al., "Prevention of Co-operative Black Hole attack in Manet on DSR protocol using Cryptographic Algorithm," International Journal of Engineering and Technology (IJET), vol/issue: 6(5), pp. 2401, 2014.

[8] A. Salh, et al., "Maximizing Energy Efficiency for Consumption Circuit Power in Downlink Massive MIMO Wireless Networks," Indonesian Journal of Electrical Engineering and Computer Science, vol/issue: 7(2), pp. 2977-2985, 2017. 